Zhuang-yu Li, Xiao-jing Liu, E. Liu, Fang-fang Jian* and Tongling Liang

\title{
Crystal structure of 1,1'-bis(diphenylphosphino)ferrocene- $\left(1,1^{\prime}\right.$-bis(diphenylphosphino)ferrocene- $\left.\mathrm{K}^{2} P, P^{\prime}\right)$-( $O$-isobutyl sulfurodithioito- $\left.\mathrm{K}^{2} S, S^{\prime}\right) \operatorname{copper}(\mathrm{I}), \mathrm{C}_{39} \mathrm{H}_{37} \mathrm{CuFeOP}_{2} \mathrm{~S}_{2}$
}

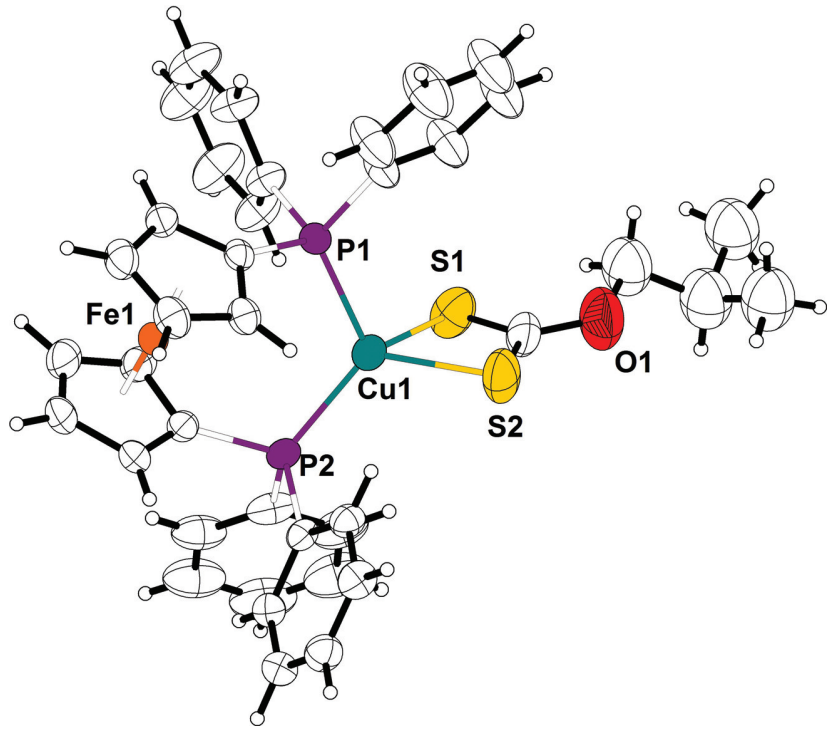

https://doi.org/10.1515/ncrs-2020-0108

Received February 24, 2020; accepted March 23, 2020; available online April 16, 2020

\section{Abstract}

$\mathrm{C}_{39} \mathrm{H}_{37} \mathrm{CuFeOP}_{2} \mathrm{~S}_{2}$, triclinic, $P \overline{1}$ (no. 2), $a=9.6132(10) \AA$, $b=11.5667(12) \AA, \quad c=16.8739(18) \AA, \quad \alpha=99.732(2)^{\circ}$, $\beta=96.342(2)^{\circ}, \quad \gamma=93.446(2)^{\circ}, \quad V=1832.0(3) \AA^{3}, \quad Z=2$, $R_{\mathrm{gt}}(F)=0.0455, w R_{\text {ref }}\left(F^{2}\right)=0.1212, T=293.0 \mathrm{~K}$.

CCDC no.: 1877712
Table 1: Data collection and handling.

\begin{tabular}{ll}
\hline Crystal: & Brownish yellow block \\
Size: & $0.25 \times 0.20 \times 0.11 \mathrm{~mm}$ \\
Wavelength: & Mo $K \alpha$ radiation $(0.71073 \AA)$ \\
$\mu:$ & $1.21 \mathrm{~mm}^{-1}$ \\
Diffractometer, scan mode: & Enraf-Nonius CAD-4, $\omega$ \\
$\theta_{\max }$, completeness: & $25.5^{\circ}, 99 \%$ \\
$N(h k l)_{\text {measured }}, N(h k l)_{\text {unique }}, R_{\text {int }}:$ & $12016,6745,0.017$ \\
Criterion for $I_{\text {obs }}, N(h k l)_{\text {gt }}:$ & $I_{\text {obs }}>2 \sigma\left(I_{\text {obs }}\right), 5281$ \\
$N(\text { param })_{\text {refined }}:$ & 442 \\
Programs: & CAD-4 [1, 2], Olex2 [3] \\
\hline
\end{tabular}

The molecular structure is shown in the figure. Table 1 contains crystallographic data and Table 2 contains the list of the atoms including atomic coordinates and displacement parameters.

\section{Source of material}

Add $O$-isobutyl $S$-hydrogen sulfurodithioite $(0.05 \mathrm{~mol})$ and copper nitrate $(0.05 \mathrm{~mol})$ to $50 \mathrm{~mL}$ of distilled water, heat the mixture and stir to reflux for $4 \mathrm{~h}$. Then add $50 \mathrm{~mL}$ DMF solution containing 1,1'-bis(diphenylphosphino)ferrocene $(0.05 \mathrm{~mol})$; heat and stir to reflux $2 \mathrm{~h}$. The reaction mixture was left to stand for 20 days, and finally brown-yellow block crystals were obtained.

\section{Experimental details}

Hydrogen atoms were placed in their geometrically idealized positions and constrained to ride on their parent atoms. The isobutyl group suffers a disorder.

\section{Comment}

Ferrocene is a metal organic complex with aromatic sandwich structure. Ferrocene and its derivatives have been widely used in catalysis [4], electrochemistry [5], functional materials [6], medicine [7], additives [8] and other fields due to their special chemical structure.

The molecular structure of the title complex is shown in the figure. Bond lengths and angles are within normal ranges. In the complex, the Cu1-P1 bond length is $2.267 \AA$ and the Cu1-P2 bond length is $2.237 \AA$. The Cu1-S1 bond length is $2.420 \AA$ and the Cu1-S2 bond length is $2.376 \AA$. The 
Table 2: Fractional atomic coordinates and isotropic or equivalent isotropic displacement parameters $\left(\AA^{2}\right)$.

\begin{tabular}{|c|c|c|c|c|}
\hline Atom & $x$ & $y$ & $z$ & $\boldsymbol{U}_{\text {iso }} * / \boldsymbol{U}_{\mathrm{eq}}$ \\
\hline Cu1 & $0.24820(4)$ & $0.55680(4)$ & $0.26054(3)$ & $0.04362(13)$ \\
\hline Fe1 & $0.59701(5)$ & $0.72905(4)$ & $0.39448(3)$ & $0.04062(14)$ \\
\hline S1 & $0.12243(12)$ & $0.49275(12)$ & $0.12634(7)$ & $0.0725(3)$ \\
\hline S2 & $0.08020(15)$ & $0.40423(12)$ & $0.27398(8)$ & $0.0816(4)$ \\
\hline P1 & $0.46810(9)$ & $0.49958(7)$ & $0.25089(5)$ & $0.0380(2)$ \\
\hline P2 & $0.24546(9)$ & $0.73535(8)$ & $0.33573(6)$ & $0.0413(2)$ \\
\hline 01 & $-0.0404(5)$ & $0.3007(4)$ & $0.1367(2)$ & $0.1123(13)$ \\
\hline $\mathrm{C} 1$ & $0.5718(6)$ & $0.2881(4)$ & $0.2865(3)$ & $0.0835(12)$ \\
\hline H1 & $0.6251(6)$ & $0.3337(4)$ & $0.3316(3)$ & $0.1002(14)^{\star}$ \\
\hline $\mathrm{C} 2$ & $0.5775(7)$ & $0.1662(4)$ & $0.2728(4)$ & $0.1003(14)$ \\
\hline $\mathrm{H} 2$ & $0.6348(7)$ & $0.1313(4)$ & $0.3086(4)$ & $0.1204(17)^{\star}$ \\
\hline C3 & $0.5012(7)$ & $0.0994(4)$ & $0.2087(4)$ & $0.0977(14)$ \\
\hline H3 & $0.5064(7)$ & $0.0183(4)$ & $0.1992(4)$ & $0.1172(16)^{\star}$ \\
\hline C4 & $0.4156(6)$ & $0.1504(4)$ & $0.1570(3)$ & $0.0925(13)$ \\
\hline H4 & $0.3619(6)$ & $0.1038(4)$ & $0.1125(3)$ & $0.1110(16)^{\star}$ \\
\hline $\mathrm{C} 5$ & $0.4082(5)$ & $0.2712(4)$ & $0.1702(3)$ & $0.0745(11)$ \\
\hline H5 & $0.3482(5)$ & $0.3049(4)$ & $0.1351(3)$ & $0.0894(13)^{\star}$ \\
\hline C6 & $0.4888(4)$ & $0.3416(3)$ & $0.2347(2)$ & $0.0548(9)$ \\
\hline $\mathrm{C} 7$ & $0.4847(4)$ & $0.6247(4)$ & $0.1260(3)$ & $0.0654(10)$ \\
\hline $\mathrm{H} 7$ & $0.3969(4)$ & $0.6474(4)$ & $0.1376(3)$ & $0.0785(12)^{\star}$ \\
\hline $\mathrm{C} 8$ & $0.5460(5)$ & $0.6674(5)$ & $0.0654(3)$ & $0.0842(12)$ \\
\hline $\mathrm{H} 8$ & $0.4994(5)$ & $0.7182(5)$ & $0.0364(3)$ & $0.1010(14)^{\star}$ \\
\hline C9 & $0.6748(5)$ & $0.6351(6)$ & $0.0482(3)$ & $0.0891(13)$ \\
\hline H9 & $0.7169(5)$ & $0.6653(6)$ & $0.0081(3)$ & $0.1069(15)^{\star}$ \\
\hline C10 & $0.7422(5)$ & $0.5589(5)$ & $0.0893(3)$ & $0.0815(12)$ \\
\hline $\mathrm{H} 10$ & $0.8296(5)$ & $0.5365(5)$ & $0.0768(3)$ & $0.0978(14)^{\star}$ \\
\hline C11 & $0.6813(4)$ & $0.5150(4)$ & $0.1493(3)$ & $0.0654(10)$ \\
\hline H11 & $0.7274(4)$ & $0.4622(4)$ & $0.1766(3)$ & $0.0785(12)^{\star}$ \\
\hline C12 & $0.5512(4)$ & $0.5490(4)$ & $0.1695(2)$ & $0.0496(8)$ \\
\hline C13 & $0.0146(5)$ & $0.8072(5)$ & $0.2510(3)$ & $0.0788(12)$ \\
\hline H13 & $-0.0180(5)$ & $0.7284(5)$ & $0.2439(3)$ & $0.0946(14)^{\star}$ \\
\hline C14 & $-0.0698(6)$ & $0.8875(6)$ & $0.2208(3)$ & $0.0995(14)$ \\
\hline H14 & $-0.1594(6)$ & $0.8632(6)$ & $0.1942(3)$ & $0.1194(17)^{\star}$ \\
\hline C15 & $-0.0189(7)$ & $1.0026(6)$ & $0.2308(3)$ & $0.0996(14)$ \\
\hline H15 & $-0.0763(7)$ & $1.0562(6)$ & $0.2115(3)$ & $0.1195(17)^{\star}$ \\
\hline C16 & $0.1127(7)$ & $1.0419(5)$ & $0.2680(3)$ & $0.0916(13)$ \\
\hline H16 & $0.1459(7)$ & $1.1202(5)$ & $0.2724(3)$ & $0.1099(16)^{\star}$ \\
\hline C17 & $0.1963(6)$ & $0.9615(4)$ & $0.2993(3)$ & $0.0722(11)$ \\
\hline H17 & $0.2859(6)$ & $0.9868(4)$ & $0.3256(3)$ & $0.0866(13)^{\star}$ \\
\hline C18 & $0.1474(4)$ & $0.8446(4)$ & $0.2917(2)$ & $0.0590(9)$ \\
\hline C19 & $0.1709(4)$ & $0.6415(3)$ & $0.4686(2)$ & $0.0455(8)$ \\
\hline H19 & $0.1988(4)$ & $0.5712(3)$ & $0.4418(2)$ & $0.0546(9)^{\star}$ \\
\hline $\mathrm{C} 20$ & $0.1266(4)$ & $0.6466(4)$ & $0.5442(2)$ & $0.0534(9)$ \\
\hline $\mathrm{H} 2 \mathrm{O}$ & $0.1260(4)$ & $0.5799(4)$ & $0.5683(2)$ & $0.0641(10)^{\star}$ \\
\hline C21 & $0.0836(4)$ & $0.7490(3)$ & $0.5840(2)$ & $0.0539(9)$ \\
\hline $\mathrm{H} 21$ & $0.0544(4)$ & $0.7518(3)$ & $0.6350(2)$ & $0.0647(10)^{\star}$ \\
\hline $\mathrm{C} 22$ & $0.0834(4)$ & $0.8479(3)$ & $0.5486(2)$ & $0.0510(8)$ \\
\hline $\mathrm{H} 22$ & $0.0529(4)$ & $0.9171(3)$ & $0.5752(2)$ & $0.0613(10)^{\star}$ \\
\hline $\mathrm{C} 23$ & $0.1286(4)$ & $0.8438(3)$ & $0.4735(2)$ & $0.0451(8)$ \\
\hline H23 & $0.1288(4)$ & $0.9109(3)$ & $0.4499(2)$ & $0.0541(9)^{\star}$ \\
\hline C24 & $0.1740(3)$ & $0.7406(3)$ & $0.4323(2)$ & $0.0393(7)$ \\
\hline C25 & $0.4186(4)$ & $0.8106(3)$ & $0.3689(2)$ & $0.0473(8)$ \\
\hline C26 & $0.4822(4)$ & $0.8543(3)$ & $0.4490(3)$ & $0.0583(9)$ \\
\hline H26 & $0.4384(4)$ & $0.8516(3)$ & $0.4985(3)$ & $0.0700(11)^{\star}$ \\
\hline
\end{tabular}

Table 2 (continued)

\begin{tabular}{|c|c|c|c|c|}
\hline Atom & $x$ & $y$ & $z$ & $U_{\text {iso }} * / U_{\text {eq }}$ \\
\hline 27 & $0.6201(5)$ & $0.9028(3)$ & $0.4454(3)$ & $0.0692(10)$ \\
\hline $\mathrm{H} 27$ & $0.6880(5)$ & $0.9393(3)$ & $0.4918(3)$ & $0.0831(12)^{\star}$ \\
\hline $\mathrm{C} 28$ & $6423(5)$ & $0.8892(3)$ & $.3634(3)$ & $0.0681(10)$ \\
\hline $\mathrm{H} 28$ & $0.7289(5)$ & $0.9139(3)$ & $0.3429(3)$ & $0.0817(12)^{\star}$ \\
\hline C29 & $0.5206(4)$ & $.8314(3)$ & $.3158(3)$ & $0.0567(9)$ \\
\hline H29 & $0.5074(4)$ & $0.8099(3)$ & $0.2566(3)$ & $0.0680(11)^{\star}$ \\
\hline C30 & $0.5497(4)$ & $0.5678(3)$ & $0.4200(2)$ & $0.0452(8)$ \\
\hline $\mathrm{H} 30$ & $0.4592(4)$ & $0.5394(3)$ & $0.4342(2)$ & $0.0543(9)^{\star}$ \\
\hline 31 & $0.6644(4)$ & $0.6252(3)$ & $0.4754(2)$ & $0.0562(9)$ \\
\hline H31 & $0.6665(4)$ & $0.6444(3)$ & $0.5344(2)$ & $0.0675(11)^{\star}$ \\
\hline C32 & $0.7739(4)$ & $0.6521(3)$ & $0.4310(3)$ & $0.0554(9)$ \\
\hline H32 & & & & 0.0665 \\
\hline C33 & $0.7294(4)$ & 0.6 & $0.3479(2)$ & $0.0455(8)$ \\
\hline H33 & 0.78 & 0.6 & $2(2)$ & $0.0546(9)^{\star}$ \\
\hline C34 & 0.58 & 0.5 & (2) & $0.0374(7)$ \\
\hline C35 & $0.0476(5)$ & 0.3946 & $0.1744(3)$ & $0.0748(12)$ \\
\hline$C 36 A^{a}$ & $-0.0522(11)$ & $0.2663(10)$ & $0.0488(4)$ & $0.115(2)$ \\
\hline $\mathrm{H} 36 \mathrm{a}^{\mathrm{a}}$ & $-0.0244(11)$ & $0.3329(10)$ & $0.0245(4)$ & $0.138(3) \star$ \\
\hline$H 36 b^{a}$ & $0.0086(11)$ & 0.2041 & & $0.138(3)^{\star}$ \\
\hline$C 37 A^{b}$ & $-0.2070(15)$ & $0.2226(13)$ & $0.0181(6)$ & $0.123(2)$ \\
\hline$H 37 A^{b}$ & $-0.2722(15)$ & $0.2846(13)$ & $0.0236(6)$ & $0.148(3)^{\star}$ \\
\hline$C 38 A^{a}$ & $-0.2475(12)$ & $0.1169(9)$ & $0.0568(6)$ & $0.131(3)$ \\
\hline$H 38 a^{a}$ & $-0.2577(12)$ & $0.1434(9)$ & $0.1128(6)$ & $0.197(4)^{\star}$ \\
\hline$H 38 b^{a}$ & $-0.3348(12)$ & $0.0782(9)$ & $0.0290(6)$ & $0.197(4)^{*}$ \\
\hline $\mathrm{H} 38 \mathrm{c}^{\mathrm{a}}$ & $-0.1755(12)$ & $0.0629(9)$ & $0.0529(6)$ & $0.197(4) *$ \\
\hline $\mathrm{C} 36 \mathrm{~B}^{\mathrm{b}}$ & $-0.107(3)$ & $0.3120(12)$ & $0.0565(9)$ & $0.130(3)$ \\
\hline$H 36 c^{b}$ & $-0.185(3)$ & $0.3607(12)$ & $0.0597(9)$ & $0.156(4) *$ \\
\hline$H 36 d^{b}$ & $-0.040(3)$ & $0.3435(12)$ & $0.0248(9)$ & $0.156(4)^{\star}$ \\
\hline$C 37 B^{a}$ & $-0.1549(12)$ & $0.1825(10)$ & $0.0221(5)$ & $0.130(2)$ \\
\hline$H 37 B^{a}$ & $-0.2283(12)$ & $0.1504(10)$ & $0.0502(5)$ & $0.156(3)^{\star}$ \\
\hline $\mathrm{C} 38 \mathrm{~B}^{\mathrm{b}}$ & $-0.025(2)$ & $0.1117(18)$ & $0.0208(16)$ & $0.136(3)$ \\
\hline$H 38 d^{b}$ & $0.016(2)$ & $0.1115(18)$ & $0.0754(16)$ & $0.205(5)^{\star}$ \\
\hline$H 38 e^{b}$ & $-0.052(2)$ & $0.0323(18)$ & $-0.0061(16)$ & $0.205(5)^{\star}$ \\
\hline$H 38 f^{b}$ & $0.042(2)$ & $0.1470(18)$ & $-0.0076(16)$ & $0.205(5)^{\star}$ \\
\hline C39 & $-0.1948(8)$ & $0.1672(6)$ & $-0.0699(4)$ & $0.1304(19)$ \\
\hline H39a & $-0.1308(8)$ & $0.2170(6)$ & $-0.0915(4)$ & $0.196(3)^{\star}$ \\
\hline $\mathrm{H} 39 \mathrm{~b}$ & $-0.2893(8)$ & & $-0.0843(4)$ & $0.196(3)^{\star}$ \\
\hline H39c & $-0.1850(8)$ & $0.0864(6)$ & $-0.0919(4)$ & $0.196(3)^{\star}$ \\
\hline
\end{tabular}

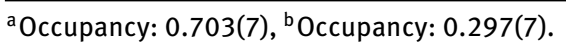

S1-Cu1-S2 bite angle is $74.6^{\circ}$ and the P1-Cu1-P2 bond angle is $113.1^{\circ}$.

Acknowledgements: The authors would like to thank the financial supports from the Henan University of Science and Technology Distinguished Professor Open Fund (Grant No. 135100001).

\section{References}

1. Enraf-Nonius. CAD-4 Software. Version 5.0. Enraf-Nonius, Delft, The Netherlands (1985). 
2. Harms, K.; Wocadlo, S.: XCAD4. University of Marburg, Germany (1995).

3. Dolomanov, O. V.; Bourhis, L. J.; Gildea, R. J.; Howard, J. A. K.; Puschmann, H.: OLEX2: a complete structure solution, refinement and analysis program. J. Appl. Crystallogr. 42 (2009) 339-341.

4. Burk, M. J.; Gross, M. F.: New chiral 1,1'-bis(phospholano) ferrocene ligands for asymmetric catalysis. Tetrahedron Lett. 35 (1994) 9363-9366.

5. Lu, D. T.; He, J.; Yu, X. Y.; Liu, X. F.; Li, Y. H.; Jiang, Z. Q.: Diiron ethanedithiolate complexes with pendant ferrocene: synthesis, characterization and electrochemistry. Polyhedron 149 (2018) $1-6$.
6. Sarhan, A. A. O.; Izumi, T.: Design and synthesis of new functional compounds related to ferrocene bearing heterocyclic moieties: a new approach towards electron donor organic materials. J. Organomet. Chem. 675 (2003) $1-12$.

7. Sijongesonke, P.; Blessing, A. A.: Ferrocene-based compounds with antimalaria/anticancer activity. Molecules 24 (2019) 3604-3604.

8. Zhang, S. Y.; Liang, B.; Fan, Y.; Wang, J. J.; Liang, X. Q.; Huang, H. F.; Huang, D.; Zhou, W. Z.; Guo, J.: Ferrocene as a novel additive to enhance the lithium-ion storage capability of $\mathrm{SnO}_{2}$ /graphene composite. ACS Appl. Mater. Interfaces 4 (2019) 31943-31953. 